\title{
Competencia cultural y población inmigrante: un reto cotidiano en nuestras consultas
}

\author{
RM. Macipe Costa ${ }^{a}$, LA. Gimeno Feliub \\ ${ }^{a}$ CS Fuentes de Ebro. Zaragoza. España. \\ ${ }^{\mathrm{b}}$ CS San Pablo. RedIAPP, Instituto Aragonés de Ciencias de la Salud. Zaragoza. España.
}

Fecha de publicación en Internet: 25 de abril de 2011

\section{Resumen}

Los últimos años, en España, ha habido un gran aumento de la población inmigrante. Este fenómeno ha supuesto un reto para los pediatras que han tenido que adaptarse a esta nueva realidad. En la actualidad, muchas de las dificultades y retos que nos plantea el niño inmigrante tienen más que ver con problemas de comunicación y diferentes concepciones de la salud y la enfermedad, que con patologías importadas o problemas de salud concretos.

En la línea de lo anterior, se habla de la competencia cultural como un conjunto de habilidades, actitudes y comportamientos, en los que el profesional debería estar entrenado para proporcionar unos cuidados sensibles, empáticos, flexibles y respetuosos con el paciente, que implican una comunicación centrada en el paciente efectiva. Son pilares básicos de la competencia cultural, la sensibilidad hacia las diferencias y hacia el otro, y la capacidad de escucha y saber preguntar.

No se trata de habilidades diferentes a las necesarias para hacer una atención adecuada a nuestros pacientes en general, sino de aplicar las habilidades necesarias para un enfoque centrado en el paciente a la población inmigrante. Por lo tanto profundizar en estas habilidades nos hará mejorar nuestra atención a la población autóctona.

En nuestra atención al inmigrante, nos encontramos con dificultades y barreras para superar estas dificultades. Dentro de estas barreras nos encontramos con problemas de actitud, prejuicios, recelos, barreras lingüísticas, escaso tiempo en las consultas, falta de formación en temas específicos de la población inmigrante, etc. Identificar estas barreras y buscar soluciones a esas dificultades son los objetivos de la presente revisión.

Palabras clave: Inmigración. Diversidad cultural. Comunicación.

Cultural competence and immigrant population: a daily challenge in our clinical offices

Abstract

In recent years, Spain has experienced a large increase in the immigrant population. This phenomenon has been a challenge for pediatricians who have had to adapt to this new reality. Today, many of the difficulties and challenges regarding immigrant children have more to do with communication problems and different conceptions of health and disease, than with imported diseases or specific health problems.

Rosa M. ${ }^{a}$ Macipe Costa, rmacipe@gmail.com

Los autores declaran no presentar conflictos de intereses en relación con la preparación y publicación de este artículo. 
According to this, we speak of cultural competence as a set of skills, attitudes and behaviours in which the practitioner should be trained in order to provide a sensitive, empathetic, flexible and patient friendly care, which also includes an effective patient-centred communication. They are cornerstones of cultural competence, together with sensitivity to differences and to others, and the ability to listen and to know how to ask.

Those abilities and skills are not different from the necessary ones to take appropriate care of our patients in general, but to apply them is necessary for a patient-centred approach to the immigrant population. Therefore the further development of these skills will improve our attention to the local population.

In our attention to immigrants we encounter difficulties and barriers to overcome. Within these barriers we face problems of attitude, prejudice, mistrust, language barriers, short time in consultations, lack of training in specific areas of the immigrant population, etc. Identifying these barriers and seeking solutions to these difficulties is the target of this review.

Key words: Immigration. Cultural diversity. Communication.

\section{Introducción}

Los últimos años, en España se ha producido un gran aumento de la población inmigrante, en su mayoría procedente de países de renta baja ${ }^{1-4}$. Este fenómeno ha llevado asociada una gran transformación de nuestra sociedad. Al contrario que hace unas décadas, España puede considerarse un país receptor de inmigración.

A fecha de 1 de enero de 2010, según datos del padrón (www.ine.es), la población inmigrante en España supone el $12 \%$ del total. Igualmente, el $12 \%$ de la población menor de 14 años es extranjera.

Somos, dentro de la Unión Europea, el país que ha recibido en los últimos años mayor número de inmigrantes. El número de residentes extranjeros se ha multiplicado por tres los últimos seis años ${ }^{5}$.
Este fenómeno ha supuesto un desafío para los pediatras, que han tenido que adaptarse a esta nueva realidad. Para ello, en los primeros años se consideró pertinente formarse en patología importada y conocer protocolos de acogida para abordar la llegada del niño inmigrante.

Pero los retos han cambiado conforme ha cambiado el momento del fenómeno. Actualmente, el flujo inmigratorio se ha estabilizado $y$, sin embargo, nacen muchos hijos de inmigrantes aquí. El abordaje de la atención a estos niños nacidos aquí y a niños que viven aquí ya hace tiempo plantea unas dificultades distintas a las que se planteaban en el momento del inicio de la inmigración. En la actualidad, muchas de las dificultades y retos que nos plantea la atención sanitaria al niño inmigrante 
tienen que ver más con problemas de comunicación y diferentes concepciones de la salud y la enfermedad, que con patologías importadas o problemas de salud concretos.

De hecho, el mismo concepto de "inmigrante" está en evolución; no es lo mismo ser inmigrante que extranjero, los niños nacidos en España de padres inmigrantes, ¿son inmigrantes?, ¿cuándo se deja de ser inmigrante?, ¿se puede llamar a los hijos de inmigrantes segunda generación de inmigrantes, cuando ellos no han sido inmigrantes? Estas cuestiones y muchas otras nos recuerdan que es un tema muy abierto y que, por lo tanto, debemos huir de conceptos e ideas cerradas.

El sistema sanitario, y sobre todo la Atención Primaria, es una de las puertas de entrada del inmigrante a nuestra sociedad. La contribución que los profesionales sanitarios podemos realizar a esta puerta de entrada es muy valiosa. La sensibilidad, la empatía y los valores interculturales de nuestra atención pueden ser uno de los medios más valiosos para favorecer la integración de la población inmigrante en nuestra sociedad.

Dotarnos de medios, herramientas $y$ capacidades para relacionarnos con personas de otras culturas es lo que nos hará profesionales competentes cultural- mente. No se trata de aprender técnicas nuevas, ni capacidades diferentes a las necesarias para relacionarnos habitualmente con el otro. El otro siempre es diferente a nosotros. Sí es cierto que percibimos al inmigrante como "más diferente" y presuponemos mayores diferencias $y$, por lo tanto, mayores dificultades en nuestra relación con él, lo cual no siempre es cierto. Probablemente, profundizar y mejorar nuestras habilidades en la relación con el inmigrante es una oportunidad que nos hará mejorar nuestra atención a las personas no inmigrantes también.

El objetivo principal de este artículo es mejorar nuestras habilidades como pediatras en el ámbito de la competencia cultural; para ello, intentaremos conocer el concepto de competencia cultural, profundizar en las barreras y dificultades que encontramos en la atención cotidiana a la población inmigrante, y proponer herramientas y soluciones a las dificultades anteriores.

\section{Definición de competencia cultural}

"La medicina de la inmigración no es una medicina de parásitos ni de virus extraños, es una medicina de hombres y mujeres que se diferencian de nosotros en la forma de expresar el sufrimiento y los problemas, en su manera de conce- 
bir la vida, las enfermedades, el dolor y la muerte. Han dejado en su país una cultura sanitaria sin haberla abandonado e incorporan otra sin haberla comprendido; es la medicina de unos enfermos que esperan de nuestro mundo sabio una tecnología mitificada que les salve, pero que no llegan a hacer comprender sus problemas interiores a sus médicos y cuidadores" (R. Colasanti. II Congreso de Medicina y Emigración. Roma, 1990).

En nuestra medicina sigue predominando un modelo biomédico en el que se hace mucho énfasis en el conocimiento de las enfermedades y se dedica poco espacio al conocimiento del enfermo ${ }^{7}$. Aspectos relativos a la relación médicopaciente, entrevista clínica, comunicación, atención a aspectos psicosociales, diferencias culturales, etc., no son suficientemente tenidos en cuenta a pesar de considerarse imprescindibles para dar una atención de calidad a nuestros pacientes.

Muchas veces diagnosticamos y tratamos a los pacientes de una forma automática, sin tener en cuenta que cada persona que tenemos delante es diferente. Sin embargo, cuando tenemos un inmigrante delante, la diferencia cultural se hace tan evidente que nos hace tomar conciencia de esas diferencias que es bueno tener en cuenta en todo acto médico?

Usualmente, el término competencia cultural hace referencia al conjunto de habilidades, actitudes y comportamientos en los que el profesional debería estar entrenado para proporcionar unos cuidados sensibles, empáticos, flexibles y respetuosos con el paciente, que implican una comunicación centrada en el paciente efectiva, que responde a las necesidades psicosociales del paciente ${ }^{8,9}$. Cuando no tenemos en cuenta las diferencias socioculturales del paciente, podemos encontrarnos con insatisfacción del paciente, problemas de adherencia y peores resultados en salud ${ }^{10}$.

El profesional sanitario debe saber atender a la población, teniendo en cuenta los aspectos diferenciales de cada uno, de manera que pueda personalizar la asistencia teniendo en cuenta la diversidad y las diferencias culturales y religiosas que influyen en la forma de entender la salud y la vida en general ${ }^{11}$.

La competencia cultural no es simplemente el estudio de las diferentes culturas, que corre el riesgo de crear estereotipos sobre la persona que tenemos delante, pues se tiende a atribuir los mismos rasgos de forma homogénea a todas las personas con una misma procedencia. Tampoco es simplemente la 
atención a las minorías étnicas? ${ }^{7}$. La habilidad no es tanto conocer en profundidad cada una de las culturas, como adquirir habilidades en torno a la interculturalidad. Parafraseando a Betancourt": "Así pues, la competencia cultural ha evolucionado desde la creación de ideas sobre los pacientes en base a la cultura, a la puesta en práctica de los principios del cuidado centrado en el paciente que incluye la empatía, la exploración de creencias, la valoración de la sensibilidad de los pacientes, sus necesidades, valores y preferencias. La competencia cultural no es una panacea que de una manera simple mejorará los resultados en salud y eliminará las desigualdades, sino un necesario repertorio de habilidades para los sanitarios que desean suministrar cuidados de alta calidad a sus pacientes".

La atención sanitaria a personas inmigradas, salvo sus peculiaridades culturales, no difiere significativamente de la que se presta a personas autóctonas. El profesional sanitario debe saber atender a la población teniendo siempre en cuenta su diversidad, y respondiendo de una forma adecuada a ella. La atención al inmigrante se simplifica cuando se incluye en el marco de una buena práctica asistencial que tiene en cuenta la distancia cultural entre médicos y pacientes en general. Según Allue ${ }^{7}$ la competencia cultural sería poco más que una sensibilidad hacia las diferencias y hacia el otro. Si se está dispuesto, sensible y culturalmente, bastarán unos pocos conocimientos referidos a la interculturalidad para que aparezca la sintonía y la confianza mutua ${ }^{12}$, pilar fundamental para una buena atención médica.

\section{Razas-culturas-personas}

En 1775, Johan Friedrich Blumenbach, publicó De generis humani varietata, donde describió cinco variedades humanas: los caucásicos (Europa, oeste de Asia, norte de África), mongólicos (este de Asia), etiópicos (África subsahariana), americanos ("Nuevo Mundo") y maláxanos (Oceanía). Estas variedades se popularizaron bajo el nombre de "blancos", "amarillos", "negros" y "rojos". Como estas divisiones fueron arbitrarias, se hicieron subdivisiones llegándose a definir entre 100 y 200 razas. La única conclusión de estos estudios raciales es que las poblaciones son diferentes, pero los límites para separar los grupos no son definidos y muchas veces son arbitrarios. Estas clasificaciones raciales son reflejo de conceptos sociales, pero no tienen una base biológica ni genética real. No tiene nada que ver una persona negra de una ciudad de Estados Unidos que una persona negra procedente del África ru- 
ral. Las grandes diferencias entre las poblaciones humanas y las barreras reales son de origen cultural y social. A su vez, todas las culturas muestran una gran diversidad interna. Incluso dentro de un mismo país y cultura no es lo mismo una persona con un nivel de estudios alto a una persona sin estudios, e incluso dentro de un mismo nivel sociocultural, todos somos distintos. Ser diferentes es algo inherente a nuestra naturaleza humana. $Y$ ser diferentes es algo bueno, que no se vive como amenazante cuando el diferente es de tu propia cultura, sino como enriquecedor ${ }^{13}$.

Así como el concepto de razas parece superado, y se enfatiza en el discurso de las culturas, también hay que revisar el discurso culturalista, que tiende a crear estereotipos, que proyectan una imagen muchas veces negativizada del paciente inmigrado, haciéndolo portador de atributos culturales estáticos y generalizados a toda la población procedente de una misma cultura ${ }^{14}$. Cuando clasificamos a un inmigrante dentro de una cultura, corremos el riesgo de estereotipar y presuponer determinadas pautas de actuación, de relación, que pueden no darse en la persona que tenemos delante. Es como cuando determinados turistas llegan a España y piensan que todos los españoles bailamos flamenco, tocamos la guitarra, vamos a los toros, vamos a misa los domingos y ayunamos los viernes de cuaresma, etc.

Entre los profesionales tendemos a buscar perfiles en personas con una misma condición común, y buscar asociaciones en nuestra práctica diaria que expliquen el comportamiento de una persona sin profundizar en las causas. Los percibimos como diferentes en su totalidad, sin plantearnos el carácter dinámico de las culturas y su adaptación a la cultura de los países de acogida. Cuando la relación se establece de forma eficaz, la experiencia nos indica que es mucho más lo que nos acerca que lo que nos aleja, e identificamos las diferencias como rasgos personales que no obstaculizan la relación ${ }^{10}$.

Con todo lo anterior, no negamos que ciertos conocimientos en patrones, características culturales, religiosas, etc., de determinados colectivos no sean útiles. Pero este conocimiento no debe llevarnos a encasillar a una persona, a presuponer comportamientos, sentimientos y pautas de actuación. Volvemos al enfoque centrado en el paciente como base de la Atención Sanitaria ${ }^{8-10}$. La persona que tenemos delante es un individuo de una determinada procedencia, con unos probables rasgos culturales que hay que verificar. Conocer e intentar comprender 
esos rasgos puede ayudarnos a empatizar con el paciente. Pero no hay que olvidar, ante todo, que lo que tenemos delante es una persona, con su carácter, su historia personal, su universo relacional, sus condiciones socioeconómicas y sus dificultades, que son suyas y no de todas las personas de una misma procedencia.

Muchas veces, cuando queremos dotarnos de habilidades interculturales, buscamos conocimientos, recetas, construidos sobre estereotipos, con los que nos gustaría obtener las claves mágicas para tratar y relacionarnos con pacientes marroquíes, africanos o chinos. Sin embargo, lo importante y necesario es situarse frente a cada paciente como un ser único, al que hay que conocer en todas sus dimensiones, y para eso lo fundamental es el saber preguntar y saber escuchar, habilidades, estas sí, fundamentales en la competencia cultural ${ }^{10}$.

Asimismo, es importante ser conscientes de que nosotros también tenemos nuestra propia cultura y que hay pautas de alimentación, comportamiento, relación, etc., que proponemos a nuestros niños, que no tienen porque ser la única o mejor manera de hacerlo. Hay que ser consciente de esto, ya que nos hará flexibles y empáticos a la hora de comprender, aconsejar y negociar con nuestros pacientes. Hay que saber diferenciar entre lo importante y demostrado científicamente, y lo que responde a comportamientos y pautas culturalmente establecidos.

\section{Barreras principales en la atención} al inmigrante

La atención al inmigrante ha supuesto un reto importante no exento de dificultades. Muchas veces, los profesionales no están preparados para lo nuevo y lo diferente, y la adaptación a esta nueva situación ha supuesto un sobreesfuerzo en unas consultas muchas veces ya muy saturadas ${ }^{15-18}$. Sin embargo, el fenómeno de la inmigración no ha hecho sino poner en evidencia unas carencias personales y del sistema para llevar adelante el enfoque biopsicosocial de la enfermedad frente a un enfoque puramente biologicista. La atención al inmigrante no exige aprender técnicas nuevas y habilidades distintas a las necesarias para hacer un enfoque centrado en el paciente?.

A pesar de esto, es evidente que existen dificultades, y existen también barreras para hacer frente a estas dificultades. Para el profesional, estas barreras pueden ser la presencia de prejuicios y estereotipos, problemas de actitud, escasa formación en medicina transcultural, problemas de comunicación, escasa 
formación en enfermedades importadas, desconocimiento en el manejo de situaciones nuevas específicas de determinadas poblaciones (ablación de clítoris, calendarios vacunales distintos, consejo al viajero, etc.).

A continuación vamos a desarrollar algunas de estas barreras.

\section{Prejuicios}

El fenómeno de la inmigración en España, a diferencia de otros países europeos, se ha caracterizado por un aumento de la población inmigrante tan rápido que no ha dado tiempo a la población a acostumbrarse y adaptarse al mismo. Esto ha generado sentimientos de amenaza y rechazo muy llamativos. Fruto de estos sentimientos son las afirmaciones que circulan en torno a la población inmigrante como, por ejemplo, que van a hundir el sistema sanitario, que abusan de él, que vienen solo a operarse, que consumen muchos mas fármacos, que saturan las urgencias, etc. A pesar de ser afirmaciones sin una base real, han tomado gran fuerza y pueden llegar a condicionar la actitud que tengamos hacia la población inmigrante. Por lo tanto, es importante estudiar y profundizar en dichas afirmaciones de cara a poder deshacer estos prejuicios.
En esa línea, la Encuesta Nacional de Salud (2006) ${ }^{19}$ ha tenido en cuenta el factor "extranjero". En dicha encuesta, aparece que el estado de salud percibido por los inmigrantes es mejor que el de los autóctonos, que tienen menos enfermedades crónicas. Asimismo, usan menos los servicios sanitarios que la población autóctona (han utilizado algún servicio sanitario en las últimas semanas el $39,5 \%$ de los autóctonos frente al $29,3 \%)^{20}$.

En los últimos años, otros estudios están ahondando en los mismos datos, confirmando la falsedad de estos tópicos, al demostrar que el uso del sistema sanitario por parte de la población inmigrante es menor que el realizado por la población autóctona ${ }^{21-26}$.

\section{Miedo a lo desconocido}

Es preciso señalar que el rechazo al extranjero es universal. El "otro" siempre ha provocado recelos, cuando no miedo. El ser humano, se dice, es un animal de costumbres, al que le cuesta salir de las rutinas establecidas, y al que toda novedad le produce una sensación de inseguridad. Decía Ortega y Gasset que el extraño, por el hecho de serlo, parece potencialmente peligroso. Cuando lo que viene de fuera resulta ser vulnerable, tiene un mayor riesgo de ser 
repudiado o apartado del grupo mayoritario $^{20}$. Vivimos en una de las sociedades más seguras que han existido jamás $y$, sin embargo, somos los que más inseguros y amenazados nos sentimos, predomina la desconfianza en los demás y sus intenciones. Y esta desconfianza es mucho mayor cuanto más diferente es el otro.

Este miedo y esta desconfianza se traducen en un deseo de separar, o alejarse de ese otro que desconocemos. Pero cuanto más se separan las personas, mayor miedo se tienen y, por lo tanto, buscan con mayor avidez la compañía de los semejantes, creándose un círculo vicioso difícil de romper. $Y$ ese deseo de evitar se transmite a los hijos, de manera que se elude llevar a los niños a escuelas en las que haya inmigrantes, creándose así escuelas ghetto, negándose a las generaciones futuras la posibilidad de aprender a convivir y normalizar la presencia de personas diferentes, que muchas veces ya ni son de otra cultura, pues han crecido dentro de la nuestra. Los extranjeros tienden a parecer más terroríficos cuanto más lejanos, desconocidos e incomprensibles los vemos.

Por otro lado, una sociedad amenaza$\mathrm{da}$, hace sentir esa amenaza y rechazo al que amenaza, y eso favorece que el rechazado se repliegue en sus iguales, fa- voreciendo asimismo el aislamiento de la sociedad de acogida, no necesitar aprender la lengua, mantener costumbres impermeables a lo nuevo, etc. En la sociedad se pueden dar redes de alta y baja densidad. Las redes de alta densidad son esas que se dan cuando todos somos iguales, nos conocemos y nos parecemos; favorecen la identidad y la rigidez de las costumbres, pues es más difícil romper con ellas cuando vives rodeado de personas que te conocen y que las comparten (por ejemplo, se dan más en las sociedades rurales). Las de baja densidad son aquellas que se dan cuando nos mezclamos con personas distintas, a las que no conocemos; favorecen la interculturalidad y el cambio (estas se dan más en las ciudades). Cuando no hay acogida, se favorecen las redes de alta densidad que, a su vez, interfieren en la integración y la interculturalidad al dificultar los cambios necesarios para irse adaptando junto a la población de acogida a un nuevo marco común.

La integración es un proceso bilateral que depende de la interacción entre el inmigrante y la sociedad receptora, y eso idealmente implica un proceso de cambio en las dos partes, cuya meta son las ganancias que ambas partes vamos a tener en el proceso más que las pérdidas. 
Existen distintos modelos de relación en el contacto de una sociedad que acoge frente al inmigrante:

- Modelo autoritario: la sociedad impone a los que llegan lo que tienen que hacer.

- Modelo de asimilación: se asume que hay diversidad pero el que llega debe ser como el que acoge, se deja atrás la cultura de la que se proviene.

- Modelo multicultural: cada uno sigue en su cultura pero sin mezclarse.

- Modelo intercultural: consiste en la creación de una cultura pública común. Vendría a ser, como dice Zygmunt Bauman, que "podemos ser diferentes y vivir juntos, y podemos aprender el arte de vivir con la diferencia, respetándola, salvaguardando la diferencia de uno y aceptando la diferencia del otro" ${ }^{13}$.

La manera de intentar dar pasos hacia esa sociedad intercultural en la que la presencia del otro se normalice, se viva como una riqueza, en la que todos aprendamos de todos, es intentar conocernos, compartir espacios y vivencias. Nosotros, como profesionales de la salud, tenemos la oportunidad de conocerlos, de acercarnos a ellos y de hacer sen- tir que la sociedad acoge, y que esa acogida facilite ese proceso de adaptación ${ }^{13}$.

A pesar de las dificultades, es un hecho que la sociedad española ha sido capaz de acoger a más de cuatro millones de inmigrados en pocos años, sin grandes problemas de convivencia. Esto es un motivo de satisfacción para el conjunto de la sociedad y un motivo de esperanza ${ }^{20}$.

\section{Comunicación}

La barrera del lenguaje es una de las mayores dificultades sentidas por los profesionales a la hora de atender a población inmigrante. Al desconocimiento de la lengua española por algunos inmigrantes se une la poca tradición española en el dominio de otras lenguas ${ }^{11,27}$.

En todos los estudios realizados, el idioma es la barrera que tanto los profesionales como la población inmigrada de lengua no hispana reconocen como la más importante en la interacción. Ciertamente, no entenderse verbalmente con una persona es un obstáculo que en ocasiones nos parece insalvable y que nos hace dudar de la beneficencia de nuestras actuaciones (por ejemplo, malentendidos en la toma de medicación). Además, un problema histórico de nuestra Atención Primaria es la falta de tiempo. Atender a una persona inmi- 
grante con la que no compartimos la lengua exige un mayor consumo del recurso "tiempo", que sentimos como escaso. Ese es un hecho que debemos valorar al organizar las actividades, y sería bueno que se contemplase en las ratios por profesional.

De todas maneras, el contacto reiterado propicia que desarrollemos habilidades personales comunicativas. El conocimiento de palabras clave y la adopción de un lenguaje no verbal adecuado generan confianza y aumentan la receptividad de la persona a la que atendemos.

En relación con la comunicación con el inmigrante, existen los siguientes principios:

- La comunicación con el inmigrante se basa en competencia cultural y técnicas de entrevista clínica.

- Sabemos que existen diferencias culturales, emocionales y a veces lingüísticas; hay que ser conscientes de ello.

- Los conocimientos, las habilidades y las actitudes en relación con la comunicación asistencial pueden aprenderse.

- El modelo de la comunicación centrada en el paciente puede ser una forma muy útil de abordaje comunicacional en la población inmigrante.
- Las técnicas de entrevista clínica que usamos en nuestras consultas pueden aplicarse a la población inmigrante teniendo en cuenta su idiosincrasia.

- La comunicación con la población inmigrante es una comunicación con la persona, como hacemos día a día con todos nuestros pacientes.

En la comunicación con el inmigrante existen unas pistas o herramientas fundamentales que vamos a comentar:

- Una buena acogida es un buen comienzo. Siempre se puede encauzar una relación pero solo hay una primera vez. Esa primera impresión es importante. Un buen comienzo es importante. Es importante que la persona sienta que es bienvenida, porque eso es fundamental en el resto de nuestra relación terapéutica.

- Es importante conectar con la persona que tenemos delante, y para ello la mirada y los gestos son fundamentales. El lenguaje no verbal es muy importante y lo descuidamos con frecuencia. En este caso, en el que el lenguaje verbal puede estar limitado, hay que intentar sacarle todo el partido posible al no verbal. 
- Es importante escuchar, y para ello es importante usar la mirada, los gestos y el cuerpo. La mirada escucha. La persona que más habilidades comunicativas tiene no es la que más habla sino la que escucha mejor. Y se escucha con la mirada y con todo el cuerpo. Nos cuesta encontrar el valor a la escucha, tendemos a hablar, a presuponer, a dar soluciones estándar; nos asusta el silencio.

- Cuando hablemos, usemos una voz cálida que transmita afecto, competencia y seguridad.

- Hay que preguntar, preguntar mucho, porque cuanto más desconocemos del otro más importante es conocer, y la forma de conocer es preguntar. Es importante hacer preguntas no muy cerradas y que no induzcan las respuestas. Ante la barrera del idioma y las interferencias culturales, tenemos tendencia a hacer preguntas con respuestas inducidas que hacen que el paciente responda lo que el entrevistador quiere o espera que responda, lo cual puede llevar a diagnósticos erróneos.

- Es preciso empatizar, intentar ponerse en el lugar del otro, y que el otro vea que estás intentando po- nerte en su lugar. Cuanto más diferentes somos, más pueden generarse sentimientos de que no nos entienden, cuando la respuesta que se da a nuestras demandas no es la que nosotros esperamos. Así que es importante usar frases que expresen que nos hemos puesto en el lugar del otro.

- Se debe usar la escucha activa. Son reglas de oro de la escucha activa:

- Dar señales no verbales de escucha (asentir, mirar, etc.).

- Dar señales verbales de escucha. Uno de los recursos de la escucha activa es parafrasear. Parafrasear es repetir una palabra o una frase de las que dice la persona con la que estamos hablando. Este recurso ayuda a sentir que estás escuchando. También es importante preguntar y resumir la información obtenida.

- No anticipar, no suponer lo que el otro va a decir.

- Antes de terminar, reformular lo más importante y comprobar que lo ha entendido.

- No tener miedo de los silencios. En el silencio hay mucha comunicación, se perciben sentimientos, ayudan a dejar pensar al usuario y 
evitar respuestas automáticas, ayudan al profesional a reflexionar sobre lo escuchado y así intervenir más adecuadamente.

- Evitar dar demasiada información. La capacidad de procesar información es limitada, si damos demasiados mensajes corremos el peligro de que el paciente no se quede con nada, y cuando hay dificultades con la lengua todavía más. Hay que intentar seleccionar lo que es imprescindible que el paciente comprenda y centrarse en eso, comprobando que lo ha entendido, haciéndole repetir por ejemplo el tratamiento propuesto.

- En nuestro caso, como pediatras, no debemos olvidar que el protagonista es el niño, con lo que es importante hablar con él, dirigirse a él, y que se sienta que forma parte de lo que está pasando en la consulta.

Todo lo anterior es siempre útil; sin embargo, en ocasiones, cuando el conocimiento de nuestra lengua del inmigrante es muy escaso, la barrera de la lengua es insalvable y necesitamos otros recursos, no siempre disponibles pero cuya existencia es bueno conocer. Todos los recursos tienen sus ventajas e inconvenientes, no existe un recurso ideal para todas las situaciones, por lo que es bueno saber cuál deberíamos usar en cada situación. Emplear un recurso u otro dependerá de la urgencia, de las necesidades y de la complejidad de lo que queremos comunicar. Dentro de estos recursos encontramos:

- Traductor. Consiste en usar una persona que nos traduzca la entrevista. Sin embargo, es un recurso con el que hay que ser cuidadoso $y$ tener en cuenta una serie de cuestiones:

- Idealmente, el traductor no debería ser alguien de la familia del paciente. Al no conocer la lengua, no tenemos control sobre lo que el traductor transmite al entrevistado. Cuando la persona que traduce no es alguien entrenado puede no entender lo que queremos preguntar y eso puede llevar a diagnósticos erróneos. Esto se agrava cuando utilizamos de traductores a los propios niños, en los que, además de agravarse este problema, se produce un cambio de roles respecto a sus padres, que puede ser motivo de conflictos intrafamiliares. No obstante, cuando queremos traducir conceptos simples puede ser útil. Tampoco hay que ol- 
vidar los posibles conflictos con la confidencialidad.

- Si el traductor es alguien de fuera de la familia, debemos pedir consentimiento al paciente y el traductor ha de saber que está obligado a la confidencialidad de lo que se hable en la consulta. El traductor debe tener claro lo que debe comunicar $y$, si no tiene claros los conceptos, debe pedir aclaraciones al profesional.

- Hay que intentar usar frases cortas y pausas frecuentes para la traducción.

- Es muy importante hablar mirando al paciente y no al traductor, tanto cuando se pregunta como cuando se escucha la respuesta.

- Hay que tener en cuenta que la presencia de una persona ajena puede impedir que el paciente cuente determinados síntomas o sentimientos; por lo tanto, aunque cueste un poco, hay que intentar la comunicación directa con el paciente, si es posible.

- La ayuda de traductores es importante cuando queremos transmitir o conocer cosas importantes, necesitamos información precisa, o lo que queremos transmitir es muy complejo.
- El traductor, a diferencia del mediador, no interviene ni opina en la relación, se limita a traducir lo más literalmente posible tanto al profesional como al paciente. Se limita a resolver las barreras lingüisticas, no las culturales, aunque a veces todo está interrelacionado.

- Existe la posibilidad de acceso a traductores por teléfono, muy útiles en situaciones de urgencia, pues normalmente podemos contar con traductores en la consulta solo de una forma programada. Hay distintas empresas que proporcionan este servicio, y hay que saber de qué servicios se dispone en cada comunidad autónoma y de la manera de acceder a ellos. Como limitaciones, tiene que, al no estar presente el traductor, no tiene información de lo que está pasando en la consulta y de toda la información no verbal presente, por lo que la información puede ser parcial.

- Mediadores:

- Resultan útiles cuando la principal barrera es la cultural, cuando vamos a abordar temas muy complejos en lo que se prevean 
conflictos y dificultades relacionadas con la cultura (ablación de clítoris, salud mental, enfermedades graves...).

- En este caso, el mediador interviene en la comunicación, no limitándose a traducir una información.

- Herramientas multilingües, traductores on-line:

- Cada vez hay más recursos online, que tienen la ventaja de que el profesional los puede usar en tiempo real, cuando se le plantea el problema, y que dan autonomía a profesional, al no depender de otra persona.

- Existen traductores sin más, pero existen también programas especializados en traducción sanitaria en los que existen frases ya hechas, que se proponen al paciente tanto en formato escrito como en audio, y el paciente responde con respuestas cerradas, por lo que son útiles sobre todo en la recogida de datos. Estos mismos programas permiten generar hojas

\begin{tabular}{|c|c|}
\hline \multirow[t]{3}{*}{ Protocolos y guías } & AEPap: www.aepap.org/inmigrante/protoc_inm.htm \\
\hline & $\begin{array}{l}\text { Guía de inmigración y salud, Gobierno de Aragón: } \\
\text { www.aragon.es (publicaciones) }\end{array}$ \\
\hline & $\begin{array}{l}\text { Manual de atención sanitaria a inmigrantes, Junta de Andalucía: } \\
\text { www.juntadeandalucia.es/salud/sites/csalud/galerias/ } \\
\text { documentos/p_4_p_2_promocion_de_la_salud/materiales_ } \\
\text { publicados_inmigrantes/manualatencioninmigrantes.pdf }\end{array}$ \\
\hline Materiales para pacientes & www.riec.es \\
\hline \multirow[t]{2}{*}{ Recursos varios } & Red ISIR: www.redisir.net \\
\hline & Salud y cultura: www.saludycultura.uji.es \\
\hline \multirow[t]{2}{*}{ Traductores } & www.universaldoctor.com \\
\hline & http://tradukka.com \\
\hline Consejos alimentación & www.naos.aesan.msps.es \\
\hline \multirow[t]{5}{*}{$\begin{array}{l}\text { Viajes Calendarios vacunales, } \\
\text { incidencias }\end{array}$} & $\begin{array}{l}\text { Ministerio de Sanidad, Política Social e Igualdad, La salud también } \\
\text { viaja: www.mspsi.es/profesionales/saludPublica/ } \\
\text { sanidadExterior/salud/home.htm }\end{array}$ \\
\hline & Viajar sano: www.viajarsano.com \\
\hline & $\begin{array}{l}\text { Consejos al viajero, Gobierno de EE. UU.: } \\
\text { http://wwwnc.cdc.gov/travel/ }\end{array}$ \\
\hline & Vacunas, AEP: www.vacunasaep.org \\
\hline & OMS, International Travel and Health: www.who.int/ith/en/ \\
\hline Otros & $\begin{array}{l}\text { Problemas sociales. Gipi: } \\
\text { http://www.infodoctor.org/gipi/ciap.htm\#H }\end{array}$ \\
\hline
\end{tabular}


de información sobre determinadas patologías, hojas de prescripción, consejos etc. Algunos de ellos aparecen en la tabla 1.

- Folletos, carteles:

- En los últimos años, debido a la presencia cada vez mayor de personas inmigrantes, se han generado documentos en varios idiomas, útiles para apoyar intervenciones.

- Existe un centro de recursos virtual en el que se puede acceder a multitud de materiales en muchos idiomas, disponible en www. riec.es.

\section{Valoración cultural}

Con las herramientas y habilidades comentadas, deberíamos ser capaces de hacer una valoración cultural del paciente inmigrante.

La valoración cultural no es únicamente una recogida de información sobre el paciente, sino un proceso que nos permite conocer a la persona, la familia y su cultura. La valoración cultural es definida por Leininger como la valoración o el examen sistemático de individuos, grupos y comunidades, así como de sus valores, creencias y prácticas, para determinar sus necesidades y las intervenciones que deben hacerse dentro de su contexto. Consiste en hacernos una idea global de la persona y su contexto cultural.

Estas diferencias y semejanzas del cuidado cultural deben ser identificadas y comprendidas para poder asistir a las personas de diferentes culturas ofreciendo un cuidado culturalmente congruente. Pretende identificar aquellas necesidades de cuidados derivadas de su situación de emigrantes: cambio de cultura, idioma, medio social, ausencia de familia, etc., así como de las derivados de sus condiciones de vida (en algunos casos): hacinamiento, malas condiciones de vivienda, tipo de alimentación, etc., e identificar los aspectos positivos hacia la salud que presentan en función de su cultura, para potenciarlos.

Así pues, serán aspectos importantes que debemos conocer:

- Procedencia y etnia de la persona.

- Datos en torno al proceso migratorio (cuándo llegó, en qué condiciones, etc.).

- Idioma principal y otras lenguas que conoce el inmigrante.

- Vivienda (cuántos viven en casa, en qué condiciones, etc.).

- Situación económica y laboral.

- Situación de regularidad o no.

- Alimentación. Existe una gran diversidad en torno a este tema en 
las distintas culturas. Las personas tendemos a mantener esos hábitos aprendidos desde que nacemos. Para nosotros como pediatras, este es un tema importante, pues acompañamos la pauta de alimentación de los niños desde que nacen. A veces, esas pautas de introducción de alimentos que proponemos son poco flexibles, a pesar de que la experiencia nos demuestra que son cambiantes, y que lo que proponíamos con rotundidad en un determinado momento luego se demuestra que no era tan importante o, incluso, que era erróneo (edad de introducción de determinados alimentos como el gluten, edad de inicio de la alimentación complementaria, dieta en la diarrea o en la hepatitis, etc.). Sin embargo, no siempre sabemos flexibilizar estas pautas e imponemos nuestro modelo de alimentación sin tener en cuenta las costumbres de las demás culturas. A veces es por convicción, otras es por desconocimiento de esos otros alimentos, por carecer de bibliografía en la que nos digan a qué edad podría introducirse ese alimento desconocido, o por otros motivos. El desconocimiento de esos otros alimentos puede hacer que los mi- nusvaloremos. En principio, no debemos convertir en un problema determinadas pautas de alimentación, mientras esos hábitos no se traduzcan en un exceso o defecto de peso, en alteraciones analíticas secundarias a esa alimentación (ferropenia) o en una alimentación claramente desequilibrada. Si esto no ocurre, no hay que interpretar una alimentación diferente a la nuestra como un problema. Hay algunas páginas Web que nos proporcionan información nutricional sobre distintos alimentos ${ }^{28,29}$ y algún documento que orienta sobre cómo conseguir una alimentación sana para todos ${ }^{30}$. Habitualmente, con el paso del tiempo la población inmigrante incorpora pautas de la alimentación del país de acogida, pero ese proceso pasa por distintas etapas de adaptación. En esta adaptación pueden incorporarse malos hábitos o alimentos poco recomendables presentes en nuestra sociedad. Nuestra función sería básicamente orientar ese proceso de adaptación hacia una alimentación sana y equilibrada. Hay que tener en cuenta también los alimentos prohibidos para determinadas religiones o culturas. No hacer de eso 
un problema si existe otro alimento permitido que reemplace el rechazado, si es necesario, para llevar una alimentación equilibrada.

- Conocimientos y cuidados que utilizaban en su país para cuidar determinadas patologías. Costumbres y creencias en torno al nacimiento, la muerte y la enfermedad. Como la población española, muchos inmigrantes utilizan remedios naturales para problemas leves de salud (diarrea, estreñimiento, resfriados, fiebre poco elevada, etc.). Aquí no disponen de dichos remedios naturales, o no saben cómo adquirirlos. Esto puede generar problemas de desatención o de consultas urgentes. Es bueno explicar poco a poco cómo resolver esos problemas leves con los medios disponibles en nuestra sociedad. Otro aspecto es valorar las creencias respecto al origen de los problemas de salud. En muchos países existen creencias que vinculan la enfermedad al "mal de ojo", y eso hace que se protejan con talismanes y amuletos, y que también protejan a sus niños con ellos. En nuestras pautas de prevención de accidentes, solemos recomendar retirar pulse- ras, colgantes etc., y deberíamos ser cuidadosos en retirar los que realmente veamos que pueden causar problemas al niño, pues la sensación de desprotección puede ser muy intensa para los padres.

- Apoyo familiar, cuidador principal, horario laboral de los padres, quién cuida a los niños cuando no están los padres. Se ha visto que los accidentes domésticos y las precipitaciones son mas frecuentes en niños inmigrantes, en ocasiones por estar los niños pequeños al cuidado de sus hermanos o sin cuidador, por eso es importante preguntar sobre este aspecto.

- Religión. Numerosas costumbres en torno al consumo de alimentos y medicamentos, y otros aspectos relacionados con la salud vienen determinados por la religión que profesan los padres.

- Expectativas de futuro. Si piensan volver a su país, si van a realizar viajes a su país, etc.

$Y$ aunque puedan existir más aspectos en los que profundizar, conocer todo lo anterior nos permitirá hacernos una buena idea del contexto sociocultural y personal que rodea a la persona que tenemos delante, y eso nos permitirá una mejor atención, una mejor aproxima- 
ción a sus problemas, una respuesta a sus demandas más próxima a sus circunstancias, lo que favorecerá la adopción de las soluciones propuestas. Si no tenemos en cuenta sus necesidades, creencias, costumbres, etc., normalmente nos encontraremos con problemas de cumplimiento importantes que al final hacen que el tiempo invertido en la intervención resulte inútil.

\section{Otros recursos}

Como decíamos al principio, la presencia de inmigrantes ha hecho que nos enfrentemos a situaciones con las que no nos habíamos encontrado anteriormente. Poco a poco se han ido respondiendo a ellas, y se han ido elaborando materiales, documentos, sitios Web, etc., que han intentado facilitarnos la labor en nuestras consultas. En la tabla 1 se indican sitios Web con recursos que vienen a responder a distintas situaciones o problemas concretos que puede plantearnos la atención a la población inmigrante. Sin duda, conocerlos aliviará en parte la sensación de estrés y sobrecarga que nos genera muchas veces la atención a esta población.

\section{Conclusiones}

La competencia cultural no es el conocimiento de las culturas, ni nos proporciona recetas concretas para abordar el trato con inmigrantes acotándolo según sus distintas procedencias. La competencia cultural viene a aplicar habilidades y técnicas necesarias e importantes en el enfoque centrado en la paciente, al caso concreto de los inmigrantes.

Son pilares básicos de la competencia cultural, la sensibilidad hacia las diferencias y hacia el otro, la capacidad de escucha y saber preguntar.

Las herramientas y actitudes que nos hacen competentes culturalmente son herramientas que harán que cualifiquemos también nuestra atención al paciente autóctono; por lo tanto, el abordaje adecuado de la persona inmigrante es una oportunidad que nos va a ayudar a mejorar nuestras habilidades de comunicación en la consulta con todos nuestros pacientes.

\section{Agradecimientos}

Agradezco a la Red ISIR (www.redisir .net) la formación y el acceso muchos de los materiales que han sido la base para la elaboración de esta revisión. 


\section{Bibliografía}

1. Berra S, Elorza-Ricart JM. Salud y uso de servicios en los sistemas sanitarios en población autóctona e inmigrante de España. Madrid: Plan de Calidad para el Sistema Nacional de Salud. Ministerio de Ciencia e Innovación. Agència d'Avaluació de Tecnologia i Recerca Mèdiques de Cataluña; 2009. Informes de Evaluación de Tecnologías Sanitarias, AATRM Núm. 2007/2008 2009.

2. Jansa JM, Garcia de Olalla P. Salud e inmigración: nuevas realidades y nuevos retos. Gac Sanit. 2004;18 Supl 1:207-13.

3. Manzardo C, Trevino B, Gomez i Prat J, Cabezos J, Mongui E, Claveria I et al. Communicable diseases in the immigrant population attended to in a tropical medicine unit: epidemiological aspects and public health issues. Travel Med Infect Dis. 2008;6(1-2):4-11.

4. Vázquez Villegas J. Inmigración y salud: ¿un nuevo modelo de atención primaria para un nuevo modelo de sociedad? Atención Primaria. 2006; 37:249.

5. Gimeno Feliu LA, Fernández de Alarcón I, Pérez E, Peñalva $D$, Ruiz-Jiménez JL, Martín E. Examen de salud a inmigrantes en Atención Primaria. Enf Emerg. 2006;8(1):40-7.

6. Malmusi D, Jansa JM, del Vallado L. Recomendaciones para la investigación e información en salud sobre definiciones y variables para el estudio de la población inmigrante de origen extranjero. Rev Esp Salud Pública. 2007;81:399409.

7. Allué X. La competencia cultural de los médicos de asistencia primaria. XV Congreso de la SVMFiC; 22-23 de mayo de 2003; Peñíscola: SVMFiC; 2003. p. 15-6.

8. Fox RC. Cultural Competence and the Culture of Medicine. N Engl J Med. 2005;353:1316-9.
9. Betancourt JR. Cultural competence-marginal or mainstream movement? N Engl J Med. 2004; 351:953-5.

10. Borrell i Carrió F. Entrevista clínica. Manual de estrategias prácticas. Barcelona: Semfyc; 2004.

11. Fuertes C, Martín Laso M. El inmigrante en la consulta de atención primaria. An Sist Sanit Navar. 2006;29(Suppl 1):9-25.

12. Martincano JL. Manual de Medicina Transcultural, 1. ${ }^{\text {a }}$ ed. Madrid: IM\&C, S.A.; 2003.

13. Bauman Z. Confianza y temor en la ciudad. Vivir con extranjeros, 6 . $^{\text {a }}$ ed. Barcelona: Atm Arcadia SL; 2009.

14. Moreno Preciado M. Del Cuidado de la Diversidad a la Diversidad del Cuidado. Index Enferm. 2006:55:7-8.

15. Esteva M, Cabrera S, Remartínez D, Díaz A, March S. Percepción de las dificultades en la atención sanitaria al inmigrante económico en medicina de familia. Aten Primaria. 2006;37(3): 154-9.

16. Martin Laso M. El paciente inmigrante en atención primaria. ¿Estamos preparados? Aten Primaria. 2001;28(2):89-90.

17. Nogués Meléndez B, Varo Gonzalo J. El médico de familia ante el fenómeno migratorio. FMC. 2004;11(Supl 3):6-11.

18. Jansà JM. Salud e inmigración, en qué punto estamos. Enf Emerg. 2006;9(2):59-60.

19. Ministerio de Sanidad y Consumo. Encuesta Nacional de Salud de España 2006 [consultado el 05/02/2011]. Disponible en www.msc.es/esta dEstudios/estadisticas/encuestaNacional/encues talndice2006.htm

20. Moreno Preciado M. Factores sociales y culturales en el cuidado del inmigrado. Cultura de los cuidados. 2008;23:5-6.

21. Gimeno Feliu LA, Armesto Gómez J, Macipe Costa R, Magallón Botaya R. Comparative study of 
paediatric prescription drug utilization between the Spanish and immigrant population. BMC Health Serv Res. 2009;9(1):225.

22. Gimeno Feliu LA, Granizo C, Sanz L, Febrel M. Inmigración y Salud. Diagnóstico de la realidad. En: Gobierno de Aragón, editor. Plan integral para la convivencia intercultural en Aragón 2008/2011. Zaragoza; 2009.

23. Rue $M$, Serna $M C$, Soler-González J, Bosch A, Ruiz-Magaz MC, Galvan L. Differences in pharmaceutical consumption and expenses between immigrant and Spanish-born populations in Lleida, (Spain): a 6-months prospective observational study. BMC Health Serv Res. 2008;8:35.

24. Regidor E, Sanz B, Pascual C, Lostao L, Sanchez E, Díaz Olalla JM. La utilización de los servicios sanitarios por la población inmigrante en España. Gac Sanit. 2009;23(Supl 1):4-11.

25. Cots F, Xastells X, Ollé C, Manzanera R, Varela J, Vall O. Perfil de la casuística hospitalaria de la población inmigrante en Barcelona. Gac Sanit. 2002;16(5):376-84.
26. Carrasco-Garrido $P$, Jiménez-García $R$, Barrera $\mathrm{VH}$, de Andrés $\mathrm{AL}$, de Miguel AG. Significant differences in the use of healthcare resources of native-born and foreign born in Spain. BMC Public Health. 2009;9:201.

27. Moreno Preciado M. La relación con el paciente inmigrante. Perspectivas investigadoras; 2005. p. 25-9.

28. What's In The Foods You Eat [en línea] [consultado el 05/02/2011]. Disponible en http:// 199.133.10.140/codesearchwebapp/\%28w1c55 q55tjol2q552lv4jgrj\%29/codesearch.aspx

29. Sociedad Española de Hipertensión. Base de datos de alimentos [en línea][consultado el 05/02/2011]. Disponible en www.seh-lelha.org/ busalimento.aspx

30. Tur Marí J, Serra Alías M, Ngo de la Cruz J, Vidal Ibáñez M. Una alimentación sana para todos. Madrid: Ministerio de Sanidad y Consumo. Estrategia NAOS; 2008 [consultado el 05/02/2011]. Disponible en www.naos.aesan.msps.es/naos/ ficheros/investigacion/informacion_nutricional_ inmigrantes.pdf 